\title{
EL REGIMEN COMUNISTA EN POLONIA DESPUES DE LA SEGUNDA GUERRA MUNDIAL
}

\author{
Jacek Golebiowski ${ }^{1}$
}

Como consecuencia de la segunda guerra mundial y de las decisiones tomadas durante las conferencias de Teherán (28 XI-1 XII 1943), Jalta (4-11 II 1945) y Potsdam (17 VII-2 VIII 1945) Polonia con los demás países de la Europa Central quedó bajo la dominación soviética. Se habían cámbiado de manera significativa las fronteras del estado polaco. Polonia había perdido a favor de la Unión Soviética sus territorios en el Este. Al mismo tiempo, como recompensa por esta perdida, había recuperado algunos territorios en el Oeste (antigua cuna de nuestro estado) perdidos como consecuencia de la colonización germánica (Drang nach Osten). El cambio de las fronteras había sido relacionado con la necesaria emigración de la población polaca de los territorios en el Este, incorporados a la Unión Soviética y de la población alemana de los territorios occidentales concedidos a Polonia (Pomerania, Silesia). Las emigraciones fueron consecuencia de las decisiones tomadas en la conferencia de Potsdam y concernían también a la población alemana en Checoslovaquia y Hungría. El nuevo territorio de Polonia tenía 312.520 kilómetros cuadrados. La frontera marítima tenía 520 kilómetros y era cuatro veces más larga que la de antes de la guerra. Según el censo de población del año 1946 Polonia contaba con 23,9 millones de habitantes.

Antes de la II guerra mundial Polonia tenía 388.000 kilómetros cuadrados y 27 millones de habitantes. Durante el periodo de entreguerras Polonia era un estado multiétnico (multinacional): se componía de polacos $-69,2 \%$; ucrania-

1. El autor, licenciado en historia, asistente en el Departamento de la Historia Contemporánea de la Universidad Católica de Lublin (Polonia). Actualmente esta preparando la tesis doctoral, Europa del Centro y Este en pensamiento político del movimiento nacionalista polaco en los años 1907-1980.

Traducción: Cezary Taracha. 
nos $-14,3 \%$; judíos $-7,8 \%$; bielorusos $-3,9 \%$ y las demás nacionalidades $-0,9 \%$. Durante la segunda República (II Rzeczpospolita) era también un país de muchas religiones y confesiones. Dominaba la Iglesia Católica, cuya confesión seguían la mayoría de los ciudadanos. Junto a la confesión romana también se profesaban las confesiones ormiana, ortodoxa y greco-católica. Teniendo en cuenta el número de creyentes, el segundo lugar lo ocupaba la Iglesia Ortodoxa y el tercero las Iglesias Protestantes. Les seguía un importante grupo de judíos y un limitado ambiente musulmán. Dicha situación cambió sustancialmente después de la II guerra mundial. En el territorio del estado polaco desde aquel momento vivieron casi exclusivamente los polacos. Desde el año de 1945, Polonia dejó de ser un país de muchas religiones y confesiones. A la Iglesia Católica pertenecía $96 \%$ de la población.

A partir de aquella fecha el pueblo polaco entró en un periodo de profundos cambios sociales y culturales impulsados desde la Unión Soviética en contextos diversos y realizados en circunstancias cambiantes (desde la extrema situación represiva en la época del estalinismo, hasta la normalización relacionada con el proceso de la descomunización).

\section{El Periodo 1945-1956}

Los años 1945-1956 son el periodo más difícil de la historia del pueblo polaco en el siglo XX. Acabada la guerra y dadas sus consecuencias, los polacos no querían consentir con el hecho de dejar su país en las manos de Stalin. La opinión pública entendía las decisiones de la Conferencia de Yalta como la traición al pueblo polaco por parte de los Aliados. Se recordaba que Polonia fue el primer país de Europa que empezó la lucha contra la Alemania de Hitler. Los soldados polacos derramaron su sangre en todos los frentes de la II Guerra Mundial. Como componentes de Fuerzas Armadas Polacas en el Occidente lucharon en Noruega, Francia, Gran Bretaña, Egipto, Libia, Palestina, Italia, Bélgica, Holanda y Alemania. En julio de 1945 el ejército polaco en la Europa Occidental contaba 211 mil soldados de tierra, 15 mil aviadores y 4,5 mil marineros. En el Este la I y la II Arma Polaca (en total contaban cerca de 400 mil soldados) participaban en los sangrientos combates contra los alemanes y en la toma de Berlín y Praga. En las tierras polacas el ejército clandestino (Armia Krajowa) organizaba acciones de diversión atacando el sistema de abastecimiento de los alemanes, de espionaje y ayudaba al pueblo oprimido por los ocupantes (también a los judíos). El ejercito clandestino movilizó durante la guerra casi 300 mil soldados.

Los datos que acabamos de referir indican la determinación de todo el pueblo polaco en la lucha contra el ocupante y en la autodefensa de su independencia. Desgraciadamente este enorme esfuerzo, las pérdidas entre la población civil y las víctimas entre los soldados, se vieron aniquilados por las 
decisiones de los Aliados. La ocupación alemana de Polonia se cambió por la ocupación soviética. El ejército polaco en el Occidente fue desmovilizado. El día 6 de julio de 1945 el gobierno británico reiteró su reconocimiento al gobierno polaco en la emigración y mandó volver a Polonia a los soldados fieles a este gobierno. La mayoría de ellos se había quedado en los países occidentales. A los que se atrevieron volver, en la nueva realidad política de Polonia, les esperaban los campos y prisiones comunistas.

Con la entrada del Ejército Rojo empezó en la República Polaca el proceso de estalinización. Se realizaba según el modelo practicado ya en la Unión Soviética. La nación polaca fue aterrorizada y paralizada. El sentimiento de incapacidad y de abandono por parte de los Aliados facilitaba la actuación de los comunistas. En realidad el poder en Polonia estaba en los manos de un órgano Ilamado Comité Polaco de Liberación Nacional (Polski Komitet Wyzwolenia Narodowego) apoyado por el Ejercito Rojo y por los servicios secretos soviéticos (NKWD).

La falta de fuerzas políticas internas que apoyaran el establecimiento del régimen comunista es una de las características de la situación en Polonia. En Polonia no hubo revolución y el llamado "nuevo orden" fue impuesto por una fuerza exterior. El nuevo aparato de poder y terror se reclutaba mayormente entre la gente de origen no polaco, enemigos de todo lo que tenía que ver con la cultura e identidad nacional polaca. Por eso las primeras víctimas del sistema estalinista fueron los representantes de la elite intelectual polaca, de la Iglesia Católica y los antiguos funcionarios del estado. Se les veía como los principales adversarios del nuevo régimen, de las nuevas condiciones sociales, instituciones y costumbres impuestas y realizadas según el modelo soviético. En los años 1944-1948 se impuso en Polonia el régimen comunista. Las más importantes etapas de este proceso fueron:

1. el establecimiento del sistema comunista mediante la dictadura del proletariado. Esto significaba en realidad la dictadura de un sólo partido comunista, totalmente ajeno, que realizaba la política prosoviética, negaba su conexión con la historia y cultura polaca y utilizaba los símbolos patrióticos solo para fines de propaganda política.

2. la reorganización del sistema del gobierno, de la administración y de la justicia según el modelo soviético

3. la reorganización del sistema industrial. Reorientación de la producción con el desarrollo de la industria pesada (sobre todo armamento) y el abandono de la industria ligera, de la que dependía el nivel de la vida de la sociedad y la situación del mercado interior polaco.

4. la destrucción de la cultura polaca a través de la imposición de las utopías del social-realismo como el único modelo de valor en el arte, en la arquitectura y la literatura y de aislar la cultura polaca de las influencias del mundo occidental. 
5. el acceso a las estructuras del poder del elemento criminal y las persecuciones de los patriotas polacos y las personas mejor preparadas para la vida pública.

6. la propaganda y realización de los más absurdos proyectos sacados de la idea de la lucha de las clases

7. la reorganización del sistema de enseñanza y educación según el modelo soviético. La falsificación de historia a través de disminuir el papel de la nación polaca y engrandecer el de la Unión Soviética.

8. la fundación de partidos políticos inspirados por la ideología marxista para cada grupo social con el fin de demostrar la pluralidad política.

9. El adoctrinamiento masivo de la sociedad con el fin de alabar el comunismo como el mejor sistema en la historia de humanidad

10. el monopolio del estado de los medios de comunicación social para la desinformación de la sociedad

11. el método de provocar los conflictos sociales ("divide et impera")

12. la promulgación de la constitución del Estado polaco basada en la de la Unión Soviética.

13. Ia lucha contra la Iglesia Católica que fue concebida por los comunistas como un factor importante de la independencia polaca y del sentido de la civilización europea.

14. absurdas reformas y colectivización de la agricultura.

15. el proceso de adoctrinamiento comunista entre los jóvenes

A pesar de las medidas tomadas y del apoyo por parte de la Unión Soviética, los comunistas que gobernaban en Polonia se daban cuenta del poco apoyo que de hecho tenían entre la sociedad. El terror masivo y la propaganda no habían podido mejorar los resultados del proceso de la comunización. Luchando contra la elite intelectual y contra la Iglesia los comunistas intentaban lograr la confianza de los obreros y campesinos. Por el decreto de 6 de XI de 1944 comenzó la reforma agraria. Después de haber realizado la reforma, el gobierno esperaba obtener el apoyo masivo por parte de los campesinos para el nuevo régimen. Para realizar la reforma se había promulgado el decreto de nacionalización de la tierra que incluía todas las parcelas más grandes de 100 hectáreas y los terrenos alemanes añadidos a Polonia después de la II Guerra Mundial. Pero el aspecto político había paralizado los efectos económicos de la reforma. Los campesinos obtenían parcelas de 5 hectáreas cada uno, lo que imposibilitaba las cuantiosas inversiones necesarias para adecuarlas a la producción para el mercado. Así la reforma agraria desencadenó efectos negativos y produjo una gran crisis en la agricultura polaca.

Para obtener el apoyo de la clase obrera el gobierno decidió ejecutar el programa de industrialización del país. Desgraciadamente en la industria también 
se seguían los modelos soviéticos (los planes trienales y quinquenales). Gracias a un enorme esfuerzo y el trabajo de millones de trabajadores se llevó a cabo el proyecto de la industrialización de Polonia cambiando su imagen de un país dominado por agricultura a un país desarrollado industrialmente. Desgraciadamente en la política económica también se perdieron muchas oportunidades por motivos políticos e ideológicos. El Ministerio de Industria controlaba totalmente no sólo las empresas estatales, sino también el sector privado, cooperativo y la artesanía. Como consecuencia del decreto de nacionalización de la industria, promulgado el día 3 de enero de 1946, se acabó con la burguesía industrial y con la libertad económica. También se habían nacionalizado todos los bancos. En 1945 el estado había conseguido ya el monopolio bancario.

Habían pasado apenas unos años después de la reforma agraria, cuando el gobierno comunista empezó el proceso de la colectivización obligando por la fuerza a los campesinos a que devolviesen sus parcelas al Estado. Los tímidos intentos de colectivización de la agricultura se interpretan como una etapa de construcción del sistema comunista y consistían en la eliminación total de la propiedad privada agraria, algo que no llegó a producirse. El proceso de industrialización y de colectivización fue acompañado por una intensa urbanización de Polonia, y en efecto se produjo la emigración masiva de los campesinos expropiados a las grandes ciudades. Entre los años 1950 y 1970 casi 6 millones de personas habían emigrado a las urbes. Así, en el año de 1970 el 52,2\% dé la población polaca vivía ya en los centros urbanos.

\section{Balance del primer decenio}

El régimen comunista de Polonia apoyado por el Ejercito Rojo y el NKWD fue visto por la nación polaca como un ocupante, contra el cual había que luchar. La actividad de la resistencia entre los años 1944 y 1948 es el mejor testimonio de esta postura. Al fin del año de 1945 las fuerzas organizadas que luchaban contra los comunistas contaban casi 100 mil personas. Esta lucha consistía en acciones de autodefensa y esporádicamente en represiones contra los funcionarios del partido comunista y de las estructuras del aparato de seguridad. Intentando comunizar Polonia los comunistas utilizaban la propaganda total y los engaños (sobre todo durante las elecciones) para legalizar su ilegal poder. Su principal intento, además de fortalecer el poder, fue el cambio de la mentalidad de la nación polaca para que viviera según las ideas de Marx y Lenin. Se creía generalmente que era el mejor camino para comunizar Polonia y eliminar las tendencias contrarias de signo nacionalista polaco. Este programa se fue realizando consecuentemente a través de la propaganda permanente $y$ el adoctrinamiento de la juventud en las escuelas y organizaciones juveniles. Pero el resultado de este programa no tuvo las consecuencias deseadas por los comunistas. Ellos no entendían los sentimientos del pueblo polaco, ni conocían su modo de pensar. Los prosoviéticos lanzaron las ideas de lucha 
de clases y el odio a la Iglesia Católica, que eran completamente ajenas a la cultura y tradición polaca. La sociedad esperaba las reformas sociales, pero se negaba aceptar en Polonia el modelo soviético de estado. Para entender esto hay que tener en cuenta que el periodo de 1918-1939 fue una época de gran desarrollo del sentimiento de la identidad nacional. La independencia recuperada después de 123 años de repartos entre las grandes potencias vecinas fue para la nación como una santidad a pesar de las diferencias políticas en la sociedad. En la defensa de esta independencia los polacos lucharon contra la Rusia de los bolcheviques en la sangrienta guerra del año 1920. Los años de la ocupación nazi hicieron más estrecha la vinculación entre varios grupos de la sociedad polaca, cohesionados también por los sentimientos religiosos. En esa situación de consenso nacionalista e interclasista, la idea de la lucha de clases no había tenido éxito. Otra vez se identificaba lo polaco con lo católico. Tampoco el enfrentamiento con la Iglesia Católica tuvo éxito. La muerte y martirio de muchos sacerdotes y la postura llena de dignidad de la Iglesia denunciando los abusos nazis durante la II Guerra Mundial hicieron que creciera su autoridad y respeto entre la sociedad polaca. En este contexto el primitivo anticlericalismo de los comunistas ofendía a los sentimientos del pueblo polaco.

El balance del primer decenio del poder fue para los comunistas muy negativo. La Iglesia Católica se mantenía como la primera fuerza moral en la nación. No llegó a concretarse en toda su amplitud el proyecto de colectivización y la tierra permaneció en las manos privadas de pequeños propietarios campesinos. La ideología marxista aparecía como una utopía que nada tenía que ver con la realidad polaca. Frente al terror comunista se consolidó la solidaridad entre los polacos. El monolito soviético llegó a romperse al intentar comunizar Polonia. Los polacos permanecieron fieles a su tradición, cultura y religión conservando la propiedad privada de la tierra e intentando sobrevivir a la época del terror estalinista. Los años de 1948-56 fueron un periodo de parálisis psíquica de la sociedad causada por el temor, de inquietudes sociales y de amargura por la traición de los Aliados. El único medicamento, la única consolación ante esta realidad, era la participación de toda la sociedad en la reconstrucción del país destruido durante la guerra.

\section{Años de 1956-1970}

La muerte de Stalin (5 III de 1953) y el XX Congreso del Partido Comunista de la Unión Soviética (14-25 II 1956) fueron momentos decisivos para todos los países comunistas. El sucesor de Stalin, Nikita Chrustchev, criticó totalmente el sistema del poder estalinista y sus errores. La crítica particularmente se centraba en el primitivo terror y sus persecuciones, el patológico culto de la persona de Stalin, la censura total de pensamiento y al abuso del concepto "enemigos del pueblo". Naturalmente la crítica tocaba sólo algunos aspectos del sistema y 
no a lo esencial del comunismo. El descrédito de los métodos estalinistas significaba el fin del periodo de terror y crímenes sancionados por la ley.

Durante los debates del famoso XX Congreso del Partido Comunista, Polonia fue representada por el primer secretario del Comité Central del partido comunista polaco (PZPR) Boleslaw Bierut. Según un comunicado oficial Bierut murió durante el Congreso de paro cardiaco. En Polonia mientras tanto se divulgaba el texto del famoso informe de Chrustchev. Se empezaba el periodo de "deshielo", la sociedad esperaba la liberalización y humanización del sistema. La esperanza de los polacos se basaba en el hecho de que Wladyslaw Gomulka fue elegido como primer secretario del partido comunista contra la voluntad de Moscú. La autonomía de Gomulka se basaba en la presión de la sociedad polaca, que a partir del año de 1956 aspiraba conscientemente a la desestalinización y democratización del sistema político. Las protestas de los obreros en la ciudad de Poznan (1956) supusieron la más grande manifestación contra los comunistas. Según ellos, la clase obrera había de ser el fundamento del partido y la fuerza que promoviera los cambios dentro de la sociedad. La resistencia de los obreros contra el partido comunista (PZPR) fue para los funcionarios políticos algo increíble, porque destruía las bases científicas del marxismo convirtiéndolo en una utopía.

Como consecuencia de las reformas de octubre de 1956 la sociedad polaca vio realizarse una parte de sus demandas. Se había disuelto el Comité de Seguridad Pública y se había disminuido el número de los funcionarios del aparato de seguridad. El desacreditado Presidente de Tribunal Supremo había dimitido. Hubo grandes cambios también en el ejército. Fueron cesados y mandados a la Unión Soviética el Ministro de Defensa, mariscal Rokossovsky y junto con él los 32 generales y coroneles rusos. Temporalmente se suspendió la práctica de las opiniones personales secretas (delaciones). Se habían cerrado los almacenes especiales y las tiendas donde hacían sus compras los miembros de la élite del partido comunista. Se había suprimido el decreto que no permitía a los ciudadanos polacos poseer oro, plata y moneda extranjera. El parlamento promulgó la ley sobre los consejos obreros - que era la base de la autonomía de los trabajadores. Muchos profesores relegados de las universidades y otros centros de enseñanza en los años anteriores volvieron a trabajar. Se había rehabilitado a los miembros de las organizaciones clandestinas que lucharon contra los nazis durante la guerra. El primado de Polonia, cardenal Stefan Wyszynski y muchos obispos encarcelados por los comunistas salieron de las prisiones.

Pero el diálogo motivado por las protestas de la sociedad y por la crisis dentro del partido comunista no duró mucho tiempo. Wladyslaw Gomulka, como los demás miembros de Comité Central de PZPR, fue consciente de que la discusión pública sobre las cuestiones políticas y económicas podía hacer comprender a la sociedad lo utópico del sistema y se pudiese llegar a negar la legitimidad del poder comunista. Por eso a finales de los años cincuenta el partido vuelve a la idea de su infalibilidad y al sistema monopartidario. El fracaso 
de las "reformas del octubre polaco" había frustrado profundamente a la sociedad polaca. En consecuencia, en los años sesenta se habla de la "pequeña estabilización", es decir la sociedad no se mezcla en la vida política y el régimen deja de profundizar en el sistema comunista. Pero mantener esta situación durante mucho tiempo era imposible. La sociedad era cada vez mas inquieta y más crítica hacia la cúpula del partido. Entre los dirigentes de PZPR se veían síntomas de discordia, inseguridad y menosprecio hacia la sociedad. La mala coyuntura económica y la falta de soluciones por parte del gobierno habían empeorado la situación.

No cabe ninguna duda de que la cuestión demográfica fue la causa de muchos problemas internos del país. El número de habitantes de Polonia creció de 24 millones en 1946 a 32,5 millones en 1970. Este crecimiento no correspondía con el desarrollo de la construcción (falta de las viviendas) y con las posibilidades de educación. Se notaba también el escaso pode adquisitivo de los salarios. La tendencia de desarrollar la industria pesado a costa de la de consumo causó la falta de los productos básicos y de alimentación y el desavastecimiento general de los mercados. El problema de la escasez de viviendas, más drástico que en los otros países comunistas, influía mucho en toda la economía nacional. No se podía resolver este problema debido a motivos ideológicos, puesto que en el modelo soviético se pensaba que la construcción de viviendas era una "inversión no productiva". Para mejorar la situación en Polonia, el régimen de Gomulka tendría que permitir el libre mercado de las viviendas. Pero el primer secretario y sus compañeros de partido no pudieron comprender las ventajas que el desarrollo del sector de la construcción podía suponer para toda economía nacional al servir como sector "locomotora" que empujara a los demás. Habían perdido la oportunidad de disminuir el paro gracias a la construcción de miles viviendas baratas y al mismo tiempo disminuir la presión del mercado de alimentación al dirigir el dinero privado hacia las viviendas. Siguiendo los principios del marxismo, los jefes del partido comunista en Polonia decidieron continuar la política de ortodoxia ideológica. Construyendo el sistema socialista no veían las auténticas necesidades y aspiraciones de la sociedad, que después de los años de guerra y las limitaciones de postguerra ya no quería vivir con carestías y desabastecimiento.

Dicha situación hizo cambiar la mentalidad de la adoctrinada clase obrera. Los trabajadores habían llegado a la conclusión de que, a pesar de ser la "clase dirigente", como les aseguraba el partido comunista, su situación económica y social no había mejorado mucho y que no tenían influencia en el poder real. Además habían advertido el enorme menosprecio que provocaban sus condiciones de vida y trabajo por parte de la elite de poder.

Los comunistas habían perdido el apoyo también de los estudiantes y del mundo universitario. Los motines estudiantiles en marzo de 1968 reprimidos por la brutal agresión de la policía y las detenciones masivas, habían desacreditado al equipo de Gomulka. El régimen había encontrado un enemigo nue- 
vo. La postura de los estudiantes sorprendió a los dirigentes, porque el acceso a la universidad dependía de determinados criterios políticos. La prioridad en el acceso la tenían los jóvenes de las familias relacionadas con el equipo de poder y de seguridad. Por otra parte, los hijos de la elite intelectual de la II República y de personas tratadas como los enemigos del comunismo no podían estudiar en las universidades.

Los motines de obreros en Gdansk, Gdynia y Szczecin, en diciembre de 1970 causaron otra crisis de poder y acabaron con el gobierno de Gomulka, el cual había perdido totalmente el contacto con la sociedad.

La Iglesia Católica mantenía una gran influencia en la situación interna de Polonia y apoyaba el rechazo del comunismo por parte de la nación polaca. Hay que subrayar sobre todo la persona del Primado de Polonia, cardenal Wyszynski que dirigió la Iglesia entre los años 1948 y 1981. Su postura llena de dignidad y santidad le facilitaron el apoyo de todo el pueblo. Como primado de Polonia supo aguantar los más primitivos ataques en la época de estalinismo e incluso la prisión en los años 1953-1956 y no permitió la confrontación abierta contra el comunismo. En su labor pastoral separaba consecuentemente el bien común de la nación y su espiritualidad católica de la vida política cotidiana. Supo evitar todas las provocaciones organizadas por los comunistas. En el año 1966, con motivo de las celebraciones del milenario del cristianismo en Polonia, preparó una gran acción pastoral, en la que las familias polacas confiaron su futuro a Jesucristo y a la Madre de Dios. Esta confesión pública de fe y el gran renacimiento de la vida espiritual del pueblo polaco confundió considerablemente a los comunistas, que no habían advertido de que continuar la lucha contra la Iglesia significaba la guerra contra toda la nación y al mismo tiempo la agonía política del comunismo.

\section{El periodo de 1970-1989}

Los años setenta en Polonia son la última oportunidad para encontrar una formula del comunismo aceptada por la sociedad. Al tener en contra a la Iglesia, los campesinos, obreros y estudiantes, los comunistas habían dejado la primitiva formula de confrontación cambiando el tono de propaganda e intentando liberalizar el sistema. El nuevo primer secretario de PZPR, Edward Gierek, era presentado en los medios de comunicación como un pragmático, un buen político y un líder abierto al dialogo con la sociedad, totalmente distinto de Gomulka, un fanático ideólogo. En la propaganda soplaban nuevos vientos, se hablaba de la "democracia socialista" y de la renovación. En este caso el régimen no quería construir un socialismo abstracto sino "una segunda Polonia" rica, un estado de bienestar y de derecho. La propaganda había dejado de utilizar motivos anticlericales. La nueva política de reconciliación con la Iglesia Católica, consistía en permitir la construcción de nuevos templos y cen- 
tros de culto, pero no duró mucho tiempo. Después de haber pasado unos años se volvió a la política de frenar todas las iniciativas eclesiásticas y a la vigilancia de los sacerdotes.

El cambio de la política económica era una indudable novedad en la actuación del equipo de Gierek. Su programa económico consistía en la aceleración de la industrialización y al mismo tiempo en construir infraestructuras de transportes, de servicios y en elevar el nivel de vida. La facilidad para obtener créditos en los países de Occidente (después del año 1973, cuándo la crisis petrolera incrementó el volumen de dinero en el mercado de inversiones) permitía realizar un programa de grandes inversiones en Polonia. El modificado plan quinquenal, aprobado por el sexto Congreso de PZPR para los años 19711975, preveía el incremento de la renta nacional en un 39\%, de la producción industrial en un $50 \%$, de la producción en agricultura en un $20 \%$, de los sueldos en un $18 \%$ y de las inversiones en un $45 \%$. La situación económica de este periodo se caracterizó por el enorme crecimiento de las inversiones. Era la consecuencia de los gigantescos créditos e importe de tecnologías de los países del Oeste (cinco veces más grande que en el quinquenio anterior). Polonia había obtenido la excepcional posibilidad de salir del atraso económico.

Se construyeron muchas industrias nuevas que devolvieron los créditos con la producción de sus primeros años. Dados los bajos gastos de producción se podía dinamizar la economía nacional. El mayor obstáculo en este proceso fueron las características del sistema económico comunista: los ineficaces mecanismos de economía planificada, el sistema de inversiones dominado por las prioridades políticas y la poca competencia de los dirigentes. Por eso ya en el año 1974 aparecieron los primeros efectos de la loca política económica. El crecimiento de la producción no estaba relacionado con el crecimiento del sistema de pagos, lo que provocó el desequilibrio en el mercado. Distintos ramos de la industria consumían mucha energía y material: el gran desperdicio y la incompetencia del personal técnico y administrativo de las grandes empresas habían aniquilado los efectos del gran esfuerzo inversor. Los problemas económicos, como consecuencia de la política del gobierno, provocaron inquietudes sociales. El gobierno intentó frenar los negativos procesos económicos subiendo los precios de la alimentación (la carne el 69\%, el azúcar el 100\%). Como reacción social, se iniciaron motines obreros en las ciudades de Ursus y Radom en el año de 1976. Otra vez la sociedad se había convencido de que el bienestar y el estado de derecho propuesto por el sistema comunista era una ficción. A partir del año 1976 empieza funcionar la oposición anticomunista. Sus principales centros fueron Varsovia, donde se formó el Comité de Defensa de los Obreros (Komitet Obrony Robotników, KOR), organizado por los representantes de elite de izquierda, y Lublín. En esta ciudad funcionaba desde el año 1918 la única, en esta parte de Europa, e independiente Universidad Católica. Los grupos relacionados con la Universidad editaban el periódico independiente "Spotkania" ("Encuentros"), por su parte KOR publicaba un "Boletín 
Informativo". Gracias a estas iniciativas por primera vez se había roto el monopolio informativo de los comunistas en Polonia. Poco a poco la actividad organizada de la oposición se desarrollaba por todo el país.

En los años setenta observamos en Polonia un gran cambio espiritual y político. Este cambio está relacionado con el hecho de que aparece una nueva generación de polacos, nacidos después del año 1956. Esta generación no conoció al terror de la época del estalinismo, no tenía miedo y estaba libre del sentimiento de resignación. Había heredado de sus padres la fe católica y la educación basada en la tradición polaca que trataba al comunismo como un cuerpo ajeno a la naturaleza polaca. Al mismo tiempo esta generación tenía mucho dinamismo, su propia visión de la política y economía y el deseo de vivir en un país normal, un estado de bienestar y de derecho. Este proceso de cambios psicológicos y políticos en Polonia fue apoyado por un acontecimiento inesperado. Se trata de la elección del arzobispo de Cracovia, cardenal Karol Wojtyla al trono papal en el año de 1978. Todos los polacos habían entendido que Polonia era un país de mucha importancia espiritual para el mundo entero. La primera visita de Juan Pablo II a Polonia (1979) había liberado una gran fuerza en la nación y el sentido de la solidaridad en la defensa de su identidad. Esta explosión de sentimientos y de fe se fortaleció gracias a las triunfales peregrinaciones del Papa por el mundo.

Desgraciadamente, al mismo tiempo, la irresponsable política del gobierno comunista hizo que la economía polaca estuviera cerca de la catástrofe. La producción de la agricultura había disminuido, se habían parado varias inversiones, dominaba la desorganización y el despilfarro. Crecía la deuda externa. La gente no podía comprar los productos más necesarios. En esta situación política se desarrolló la actividad de la oposición. Muchas publicaciones sin censurar informaban a la sociedad polaca sobre el estado efectivo del estado y de la economía. Las acciones de la oposición provocaron la represión del gobierno.

A finales de los años setenta había cambiado la mentalidad de la sociedad. Este cambio estaba relacionado con la política del régimen, con la crisis económico-social y con la enseñanza de la Iglesia sobre la necesidad de vivir en la verdad y de valorar la dignidad de la persona humana. En consecuencia todas las fuerzas patrióticas se movilizaron con un solo fin, la defensa contra la destrucción comunista. En julio de 1980 empezó una ola de protestas y huelgas contra la política del gobierno. En agosto la huelga general paralizó todo el país. Fue la más grande huelga contra el comunismo én la Europa del Este.

Resumiendo los años setenta en Polonia hay que decir que este periodo fue el menos represivo en toda la etapa comunista de nuestro país. Muchas familias polacas disfrutaron de la prosperidad de los años 1970-1975 haciendo inversiones en viviendas, renovando sus haciendas y propiedades. Se había modernizado la agricultura polaca y las empresas agrarias. Otra característica de aquella época fue la llegada al poder del gobierno de Gierek. En este equi- 
po no había lugar para los ideólogos fervientes del comunismo. En el partido comunista triunfaron las personas que orientaban su carrera política hacia el pragmatismo, como medio para llegar al poder o al dinero. Los contactos de los comunistas polacos con Occidente en los años setenta fortalecieron estas tendencias y el deseo de vivir según los modelos típicos de la Europa del Oeste.

La fundación del movimiento sindical "Solidaridad" en el año 1980 y el periodo de libertad (desde agosto de 1980 hasta el estado de emergencia de 13 de diciembre de 1981) se pueden tratar como una gran conclusión de los cambios de los años setenta en Polonia. Aquellos quince meses de vida en la patria libre habían dado lugar a otros cambios que ya no se podían parar. La verdad sobre la situación del país, de la economía y la herencia de los gobiernos comunistas, conocida hasta el momento sólo por determinados ambientes de la oposición, se había divulgado a toda la sociedad.

El comunismo, como doctrina y sistema socio-político se había desacreditado totalmente. En el año de 1980 se habían terminado en Polonia las pruebas para reformar el comunismo. Su persistencia hasta el año 1989 fue relacionada con la situación internacional. "Los gobiernos de los ancianos" en el Kremlín intentaban parar los inevitables procesos de descomunización en Polonia y en toda Europa Central. El estado de emergencia en Polonia (1981) hizo profundizar las diferencias de la sociedad polaca entre los perseguidores y sus víctimas. Fue una época de resignación y de pesar por haber perdido la oportunidad de reformar el país. Se habían malogrado los mejores años de entusiasmo nacional en el siglo $\mathrm{XX}$, para construir un país moderno y un estado de derecho.

\section{Bibliografia}

Prensa: "Myœl Polska" (Londres); "Opoka" (Londres) y "Horyzonty" (ParísLondrs-Nueva York)

Albert A., Historia Polski 1918-1980, Londres 1989.

Bromke A., Pañstwa komunistyczne, Nueva York 1975.

BRZEZINSKI Z., Jednoœeæ czy konflikty, Nueva York 1978.

HIRSZ Z., Historia polityczna Polski, Bialystok 1996.

Historia Europy, praca zbiorowa, Wroc3aw 1998.

KarPINSKI J., Polska, komunizm, opozycja, Londres 1988.

KERSTEN K., Narodziny systemu w3adzy, Varsovia 1992.

KERSTEN K., Jalta, Varsovia 1990.

KONOPCZYNSKI W., Historia polityczna Polski 1914-1939, Varsovia 1995.

Laqueur W., Historia Europy 1945-1992, Londyn 1993.

Roszkowski W., Historia Polski 1918-1990, Varsovia 1995.

Roszkowskı W., Historia polityczna swiata po 1945 roku, Varsovia 1997. 